\title{
Complex left Caputo fractional inequalities
}

\author{
George A. Anastassiou
}

\begin{abstract}
Here we present several complex left Caputo type fractional inequalities of well known kinds, such as of Ostrowski, Poincare, Sobolev, Opial and HilbertPachpatte.
\end{abstract}

Mathematics Subject Classification (2010): 26D10, 26D15, 30A10.

Keywords: Complex inequalities, fractional inequalities, Caputo fractional derivative.

\section{Introduction}

We are motivated by the following result for functions of complex variable: Complex Ostrowski type inequality

Theorem 1.1. (see [3]) Let $f$ be holomorphic in $G$, an open domain and suppose $\gamma \subset G$ is a smooth path from $z(a)=u$ to $z(b)=w$. If $v=z(x)$ with $x \in(a, b)$, then $\gamma_{u, w}=\gamma_{u, v} \cup \gamma_{v, w}$,

$$
\begin{aligned}
\left|f(v)(w-u)-\int_{\gamma} f(z) d z\right| & \leq\left\|f^{\prime}\right\|_{\gamma_{u, v} ; \infty} \int_{\gamma_{u, v}}|z-u||d z| \\
& +\left\|f^{\prime}\right\|_{\gamma_{v, w} ; \infty} \int_{\gamma_{v, w}}|z-w||d z| \\
& \leq\left[\int_{\gamma_{u, v}}|z-u||d z|+\int_{\gamma_{v, w}}|z-w||d z|\right]\left\|f^{\prime}\right\|_{\gamma_{u, w} ; \infty},
\end{aligned}
$$

and

$$
\begin{aligned}
\left|f(v)(w-u)-\int_{\gamma} f(z) d z\right| & \leq \max _{z \in \gamma_{u, v}}|z-u|\left\|f^{\prime}\right\|_{\gamma_{u, v} ; 1}+\max _{z \in \gamma_{v, w}}|z-w|\left\|f^{\prime}\right\|_{\gamma_{v, w} ; 1} \\
& \leq \max \left\{\max _{z \in \gamma_{u, v}}|z-u|, \max _{z \in \gamma_{v, w}}|z-w|\right\}\left\|f^{\prime}\right\|_{\gamma_{u, w} ; 1} .
\end{aligned}
$$


If $p, q>1$ with $\frac{1}{p}+\frac{1}{q}=1$, then

$$
\begin{aligned}
\left|f(v)(w-u)-\int_{\gamma} f(z) d z\right| & \leq\left(\int_{\gamma_{u, v}}|z-u|^{q}|d z|\right)^{\frac{1}{q}}\left\|f^{\prime}\right\|_{\gamma_{u, v} ; p} \\
& +\left(\int_{\gamma_{v, w}}|z-w|^{q}|d z|\right)^{\frac{1}{q}}\left\|f^{\prime}\right\|_{\gamma_{v, w} ; p} \\
& \leq\left(\int_{\gamma_{u, v}}|z-u|^{q}|d z|+\int_{\gamma_{v, w}}|z-w|^{q}|d z|\right)^{\frac{1}{q}}\left\|f^{\prime}\right\|_{\gamma_{u, w} ; p} .
\end{aligned}
$$

Above $|\cdot|$ is the complex absolute value.

We are also motivated by the next complex Opial type inequality:

Theorem 1.2. (see [2]) Let $f: D \subseteq \mathbb{C} \rightarrow \mathbb{C}$ be an analytic function on the domain $D$ and let $x, y, w \in D$. Suppose $\gamma$ is a smooth path parametrized by $z(t), t \in[a, b]$ with $z(a)=x, z(c)=y$, and $z(b)=w$, where $c \in[a, b]$ is floating. Assume that $f^{(k)}(x)=0, k=0,1, \ldots, n, n \in \mathbb{Z}_{+}$, and $p, q>1: \frac{1}{p}+\frac{1}{q}=1$. Then

1)

$$
\begin{aligned}
& \left|\int_{a}^{b} f(z(t)) f^{(n+1)}(z(t)) z^{\prime}(t) d t\right| \\
\leq & \int_{a}^{b}|f(z(t))|\left|f^{(n+1)}(z(t))\right|\left|z^{\prime}(t)\right| d t \\
\leq & \frac{1}{2^{\frac{1}{q}} n !}\left[\int_{a}^{b}\left(\int_{a}^{c}|z(c)-z(t)|^{p n}\left|z^{\prime}(t)\right| d t\right)\left|z^{\prime}(c)\right| d c\right]^{\frac{1}{p}} \\
\cdot & \left(\int_{a}^{b}\left|f^{(n+1)}(z(t))\right|^{q}\left|z^{\prime}(t)\right| d t\right)^{\frac{2}{q}},
\end{aligned}
$$

equivalently it holds

2)

$$
\begin{gathered}
\left|\int_{\gamma_{x, w}} f(z) f^{(n+1)}(z) d z\right| \leq \int_{\gamma_{x, w}}|f(z)|\left|f^{(n+1)}(z)\right||d z| \\
\leq \frac{1}{2^{\frac{1}{q}} n !}\left[\int_{a}^{b}\left(\int_{\gamma_{x, y}}|z(c)-z|^{p n}|d z|\right)\left|z^{\prime}(c)\right| d c\right]^{\frac{1}{p}}\left(\int_{\gamma_{x, w}}\left|f^{(n+1)}(z)\right|^{q}|d z|\right)^{\frac{2}{q}} .
\end{gathered}
$$

Here we utilize on $\mathbb{C}$ the results of [1] which are for general Banach space valued functions.

Mainly we give different cases of the left fractional $\mathbb{C}$-Ostrowski type inequality and we continue with the left fractional: $\mathbb{C}$-Poincaré like and Sobolev like inequalities.

We present an Opial type left $\mathbb{C}$-fractional inequality, and we finish with the Hilbert-Pachpatte left $\mathbb{C}$-fractional inequalities. 


\section{Background}

In this section all integrals are of Bochner type.

We need

Definition 2.1. (see [4]) A definition of the Hausdorff measure $h_{\alpha}$ goes as follows: if $(T, d)$ is a metric space, $A \subseteq T$ and $\delta>0$, let $\Lambda(A, \delta)$ be the set of all arbitrary collections $(C)_{i}$ of subsets of $T$, such that $A \subseteq \cup_{i} C_{i}$ and $\operatorname{diam}\left(C_{i}\right) \leq \delta$ (diam =diameter) for every $i$. Now, for every $\alpha>0$ define

$$
h_{\alpha}^{\delta}(A):=\inf \left\{\sum\left(\operatorname{diam} C_{i}\right)^{\alpha} \mid\left(C_{i}\right)_{i} \in \Lambda(A, \delta)\right\} .
$$

Then there exists $\lim _{\delta \rightarrow 0} h_{\alpha}^{\delta}(A)=\sup _{\delta>0} h_{\alpha}^{\delta}(A)$, and $h_{\alpha}(A):=\lim _{\delta \rightarrow 0} h_{\alpha}^{\delta}(A)$ gives an outer measure on the power set $\mathcal{P}(T)$, which is countably additive on the $\sigma$-field of all Borel subsets of $T$. If $T=\mathbb{R}^{n}$, then the Hausdorff measure $h_{n}$, restricted to the $\sigma$-field of the Borel subsets of $\mathbb{R}^{n}$, equals the Lebesgue measure on $\mathbb{R}^{n}$ up to a constant multiple. In particular, $h_{1}(C)=\mu(C)$ for every Borel set $C \subseteq \mathbb{R}$, where $\mu$ is the Lebesgue measure.

Definition 2.2. ([1]) Let $[a, b] \subset \mathbb{R}, X$ be a Banach space, $\nu>0 ; n:=\lceil\nu\rceil \in \mathbb{N},\lceil\cdot\rceil$ is the ceiling of the number, $f:[a, b] \rightarrow X$. We assume that $f^{(n)} \in L_{1}([a, b], X)$. We call the Caputo-Bochner left fractional derivative of order $\nu$ :

$$
\left(D_{* a}^{\nu} f\right)(x):=\frac{1}{\Gamma(n-\nu)} \int_{a}^{x}(x-t)^{n-\nu-1} f^{(n)}(t) d t, \quad \forall x \in[a, b] .
$$

If $\nu \in \mathbb{N}$, we set $D_{* a}^{\nu} f:=f^{(\nu)}$ the ordinary $X$-valued derivative, defined similarly to the numerical one, and also set $D_{* a}^{0} f:=f$.

By [1] $\left(D_{* a}^{\nu} f\right)(x)$ exists almost everywhere in $x \in[a, b]$ and $D_{* a}^{\nu} f \in L_{1}([a, b], X)$. If $\left\|f^{(n)}\right\|_{L_{\infty}([a, b], X)}<\infty$, then by [1] $D_{* a}^{\nu} f \in C([a, b], X)$.

We need the left-fractional Taylor's formula:

Theorem 2.3. ([1]) Let $n \in \mathbb{N}$ and $f \in C^{n-1}([a, b], X)$, where $[a, b] \subset \mathbb{R}$ and $X$ is $a$ Banach space, and let $\nu \geq 0: n=\lceil\nu\rceil$. Set

$$
F_{x}(t):=\sum_{i=0}^{n-1} \frac{(x-t)^{i}}{i !} f^{(i)}(t), \quad \forall t \in[a, x],
$$

where $x \in[a, b]$.

Assume that $f^{(n)}$ exists outside a $\mu$-null Borel set $B_{x} \subseteq[a, x]$, such that

$$
h_{1}\left(F_{x}\left(B_{x}\right)\right)=0, \forall x \in[a, b] .
$$

We also assume that $f^{(n)} \in L_{1}([a, b], X)$. Then

$$
f(x)=\sum_{i=0}^{n-1} \frac{(x-a)^{i}}{i !} f^{(i)}(a)+\frac{1}{\Gamma(\nu)} \int_{a}^{x}(x-z)^{\nu-1}\left(D_{* a}^{\nu} f\right)(z) d z,
$$

$\forall x \in[a, b]$. 
Next we mention an Ostrowski type inequality at left fractional level for Banach valued functions.

Theorem 2.4. ([1]) Let $\nu \geq 0, n=\lceil\nu\rceil$. Here all as in Theorem 2.3. Assume that $f^{(i)}(a)=0, i=1, \ldots, n-1$, and that $D_{* a}^{\nu} f \in L_{\infty}([a, b], X)$. Then

$$
\left\|\frac{1}{b-a} \int_{a}^{b} f(x) d x-f(a)\right\| \leq \frac{\left\|D_{* a}^{\nu} f\right\|_{L_{\infty}([a, b], X)}}{\Gamma(\nu+2)}(b-a)^{\nu} .
$$

We mention an Ostrowski type $L_{p}$ fractional inequality:

Theorem 2.5. ([1]) Let $p, q>1: \frac{1}{p}+\frac{1}{q}=1$, and $\nu>\frac{1}{q}, n=\lceil\nu\rceil$. Here all as in Theorem 2.3. Assume that $f^{(k)}(a)=0, k=1, \ldots, n-1$, and $D_{* a}^{\nu} f \in L_{q}([a, b], X)$, where $X$ is a Banach space. Then

$$
\left\|\frac{1}{b-a} \int_{a}^{b} f(x) d x-f(a)\right\| \leq \frac{\left\|D_{* a}^{\nu} f\right\|_{L_{q}([a, b], X)}}{\Gamma(\nu)(p(\nu-1)+1)^{\frac{1}{p}}\left(\nu+\frac{1}{p}\right)}(b-a)^{\nu-\frac{1}{q}} .
$$

It follows

Corollary 2.6. ([1]) (to Theorem 2.5, case of $p=q=2$ ). Let $\nu>\frac{1}{2}, n=\lceil\nu\rceil$. Here all as in Theorem 2.3. Assume that $f^{(k)}(a)=0, k=1, \ldots, n-1$, and $D_{* a}^{\nu} f \in$ $L_{2}([a, b], X)$. Then

$$
\left\|\frac{1}{b-a} \int_{a}^{b} f(x) d x-f(a)\right\| \leq \frac{\left\|D_{* a}^{\nu} f\right\|_{L_{2}([a, b], X)}}{\Gamma(\nu)(\sqrt{2 \nu-1})\left(\nu+\frac{1}{2}\right)}(b-a)^{\nu-\frac{1}{2}} .
$$

Next comes the $L_{1}$ case of fractional Ostrowski inequality:

Theorem 2.7. ([1]) Let $\nu \geq 1, n=\lceil\nu\rceil$, and all as in Theorem 2.3. Assume that $f^{(k)}(a)=0, k=1, \ldots, n-1$, and $D_{* a}^{\nu} f \in L_{1}([a, b], X)$. Then

$$
\left\|\frac{1}{b-a} \int_{a}^{b} f(x) d x-f(a)\right\| \leq \frac{\left\|D_{* a}^{\nu} f\right\|_{L_{1}([a, b], X)}}{\Gamma(\nu+1)}(b-a)^{\nu-1} .
$$

We continue with a Poincaré like fractional inequality:

Theorem 2.8. ([1]) Let $p, q>1: \frac{1}{p}+\frac{1}{q}=1$, and $\nu>\frac{1}{q}, n=\lceil\nu\rceil$. Here all as in Theorem 2.3. Assume that $f^{(k)}(a)=0, k=0,1, \ldots, n-1$, and $D_{* a}^{\nu} f \in L_{q}([a, b], X)$, where $X$ is a Banach space. Then

$$
\|f\|_{L_{q}([a, b], X)} \leq \frac{(b-a)^{\nu}}{\Gamma(\nu)(p(\nu-1)+1)^{\frac{1}{p}}(q \nu)^{\frac{1}{q}}}\left\|D_{* a}^{\nu} f\right\|_{L_{q}([a, b], X)} .
$$

Next comes a Sobolev like fractional inequality.

Theorem 2.9. ([1]) All as in the last Theorem 2.8. Let $r>0$. Then

$$
\|f\|_{L_{r}([a, b], X)} \leq \frac{(b-a)^{\nu-\frac{1}{q}+\frac{1}{r}}}{\Gamma(\nu)(p(\nu-1)+1)^{\frac{1}{p}}\left(r\left(\nu-\frac{1}{q}\right)+1\right)^{\frac{1}{r}}}\left\|D_{* a}^{\nu} f\right\|_{L_{q}([a, b], X)} .
$$


We mention the following Opial type fractional inequality:

Theorem 2.10. ([1]) Let $p, q>1: \frac{1}{p}+\frac{1}{q}=1$, and $\nu>\frac{1}{q}, n:=\lceil\nu\rceil$. Let $[a, b] \subset \mathbb{R}, X$ a Banach space, and $f \in C^{n-1}([a, b], X)$. Set

$$
F_{x}(t):=\sum_{i=0}^{n-1} \frac{(x-t)^{i}}{i !} f^{(i)}(t), \forall t \in[a, x], \text { where } x \in[a, b] .
$$

Assume that $f^{(n)}$ exists outside a $\mu$-null Borel set $B_{x} \subseteq[a, x]$, such that

$$
h_{1}\left(F_{x}\left(B_{x}\right)\right)=0, \forall x \in[a, b] .
$$

We also assume that $f^{(n)} \in L_{\infty}([a, b], X)$.

Assume also that $f^{(k)}(a)=0, k=0,1, \ldots, n-1$. Then

$$
\begin{gathered}
\int_{a}^{x}\|f(w)\|\left\|\left(D_{* a}^{\nu} f\right)(w)\right\| d w \\
\leq \frac{(x-a)^{\nu-1+\frac{2}{p}}}{2^{\frac{1}{q}} \Gamma(\nu)((p(\nu-1)+1)(p(\nu-1)+2))^{\frac{1}{p}}}\left(\int_{a}^{x}\left\|\left(D_{* a}^{\nu} f\right)(z)\right\|^{q} d z\right)^{\frac{2}{q}}, \\
\forall x \in[a, b] .
\end{gathered}
$$

We finish this section with a Hilbert-Pachpatte left fractional inequality:

Theorem 2.11. ([1]) Let $p, q>1: \frac{1}{p}+\frac{1}{q}=1$, and $\nu_{1}>\frac{1}{q}, \nu_{2}>\frac{1}{p}, n_{i}:=\left\lceil\nu_{i}\right\rceil, i=1,2$. Here $\left[a_{i}, b_{i}\right] \subset \mathbb{R}, i=1,2 ; X$ is a Banach space. Let $f_{i} \in C^{n_{i}-1}\left(\left[a_{i}, b_{i}\right], X\right), i=1,2$. Set

$$
F_{x_{i}}\left(t_{i}\right):=\sum_{j_{i}=0}^{n_{i}-1} \frac{\left(x_{i}-t_{i}\right)^{j_{i}}}{j_{i} !} f_{i}^{\left(j_{i}\right)}\left(t_{i}\right)
$$

$\forall t_{i} \in\left[a_{i}, x_{i}\right]$, where $x_{i} \in\left[a_{i}, b_{i}\right] ; i=1,2$. Assume that $f_{i}^{\left(n_{i}\right)}$ exists outside a $\mu$-null Borel set $B_{x_{i}} \subseteq\left[a_{i}, x_{i}\right]$, such that

$$
h_{1}\left(F_{x_{i}}\left(B_{x_{i}}\right)\right)=0, \forall x_{i} \in\left[a_{i}, b_{i}\right] ; i=1,2 .
$$

We also assume that $f_{i}^{\left(n_{i}\right)} \in L_{1}\left(\left[a_{i}, b_{i}\right], X\right)$, and

$$
f_{i}^{\left(k_{i}\right)}\left(a_{i}\right)=0, \quad k_{i}=0,1, \ldots, n_{i}-1 ; i=1,2,
$$

and

$$
\left(D_{* a_{1}}^{\nu_{1}} f_{1}\right) \in L_{q}\left(\left[a_{1}, b_{1}\right], X\right), \quad\left(D_{* a_{2}}^{\nu_{2}} f_{2}\right) \in L_{p}\left(\left[a_{2}, b_{2}\right], X\right)
$$

Then

$$
\begin{gathered}
\int_{a_{1}}^{b_{1}} \int_{a_{2}}^{b_{2}} \frac{\left\|f_{1}\left(x_{1}\right)\right\|\left\|f_{2}\left(x_{2}\right)\right\| d x_{1} d x_{2}}{\left(\frac{\left(x_{1}-a_{1}\right)^{p\left(\nu_{1}-1\right)+1}}{p\left(p\left(\nu_{1}-1\right)+1\right)}+\frac{\left(x_{2}-a_{2}\right)^{q\left(\nu_{2}-1\right)+1}}{q\left(q\left(\nu_{2}-1\right)+1\right)}\right)} \\
\leq \frac{\left(b_{1}-a_{1}\right)\left(b_{2}-a_{2}\right)}{\Gamma\left(\nu_{1}\right) \Gamma\left(\nu_{2}\right)}\left\|D_{* a_{1}}^{\nu_{1}} f_{1}\right\|_{L_{q}\left(\left[a_{1}, b_{1}\right], X\right)}\left\|D_{* a_{2}}^{\nu_{2}} f_{2}\right\|_{L_{p}\left(\left[a_{2}, b_{2}\right], X\right)} .
\end{gathered}
$$




\section{Main results}

We need a special case of Definition 2.2 over $\mathbb{C}$.

Definition 3.1. Let $[a, b] \subset \mathbb{R}, \nu>0 ; n:=\lceil\nu\rceil \in \mathbb{N},\lceil\cdot\rceil$ is the ceiling of the number and $f \in C^{n}([a, b], \mathbb{C})$. We call Caputo-Complex left fractional derivative of order $\nu$ :

$$
\left(D_{* a}^{\nu} f\right)(x):=\frac{1}{\Gamma(n-\nu)} \int_{a}^{x}(x-t)^{n-\nu-1} f^{(n)}(t) d t, \quad \forall x \in[a, b],
$$

where the derivatives $f^{\prime}, \ldots f^{(n)}$ are defined as the numerical derivative.

If $\nu \in \mathbb{N}$, we set $D_{* a}^{\nu} f:=f^{(\nu)}$ the ordinary $\mathbb{C}$-valued derivative and also set $D_{* a}^{0} f:=f$.

Notice here (by [1]) that $D_{* a}^{\nu} f \in C([a, b], \mathbb{C})$.

We make

Remark 3.2. Suppose $\gamma$ is a smooth path parametrized by $z(t), t \in[a, b]$ (i.e. there exists $z^{\prime}(t)$ and is continuous) and from now on $f$ is a complex function which is continuous on $\gamma$.

Put $z(a)=u$ and $z(b)=w$ with $u, w \in \mathbb{C}$. We define the integral of $f$ on $\gamma_{u, w}=\gamma$ as

$$
\int_{\gamma} f(z) d z=\int_{\gamma_{u, w}} f(z) d z:=\int_{a}^{b} f(z(t)) z^{\prime}(t) d t=\int_{a}^{b} h(t) d t,
$$

where $h(t):=f(z(t)) z^{\prime}(t), t \in[a, b]$.

We notice that the actual choice of parametrization of $\gamma$ does not matter.

This definition immediately extends to paths that are piecewise smooth. Suppose $\gamma$ is parametrized by $z(t), t \in[a, b]$, which is differentiable on the intervals $[a, c]$ and $[c, b]$, then assuming that $f$ is continuous on $\gamma$ we define

$$
\int_{\gamma_{u, w}} f(z) d z:=\int_{\gamma_{u, v}} f(z) d z+\int_{\gamma_{v, w}} f(z) d z,
$$

where $v:=z(c)$. This can be extended for a finite number of intervals.

We also define the integral with respect to arc-length

$$
\int_{\gamma_{u, w}} f(z)|d z|:=\int_{a}^{b} f(z(t))\left|z^{\prime}(t)\right| d t
$$

and the length of the curve $\gamma$ is then

$$
l(\gamma)=\int_{\gamma_{u, w}}|d z|:=\int_{a}^{b}\left|z^{\prime}(t)\right| d t
$$

We mention also the triangle inequality for the complex integral, namely

$$
\left|\int_{\gamma} f(z) d z\right| \leq \int_{\gamma}|f(z)||d z| \leq\|f\|_{\gamma, \infty} l(\gamma)
$$

where $\|f\|_{\gamma, \infty}:=\sup _{z \in \gamma}|f(z)|$.

We give the following left-fractional $\mathbb{C}$-Taylor's formula: 
Theorem 3.3. Let $h \in C^{n}([a, b], \mathbb{C}), n=\lceil\nu\rceil, \nu \geq 0$. Then

$$
h(t)=\sum_{i=0}^{n-1} \frac{(t-a)^{i}}{i !} h^{(i)}(a)+\frac{1}{\Gamma(\nu)} \int_{a}^{t}(t-\lambda)^{\nu-1}\left(D_{* a}^{\nu} h\right)(\lambda) d \lambda,
$$

$\forall t \in[a, b]$, in particular it holds,

$$
\begin{aligned}
f(z(t)) z^{\prime}(t) & =\sum_{i=0}^{n-1} \frac{(t-a)^{i}}{i !}\left(f(z(a)) z^{\prime}(a)\right)^{(i)} \\
& +\frac{1}{\Gamma(\nu)} \int_{a}^{t}(t-\lambda)^{\nu-1}\left(D_{* a}^{\nu} f(z(\cdot)) z^{\prime}(\cdot)\right)(\lambda) d \lambda,
\end{aligned}
$$

$\forall t \in[a, b]$.

Proof. By Theorem 2.3.

It follows a left fractional $\mathbb{C}$-Ostroswski type inequality

Theorem 3.4. Let $n \in \mathbb{N}$ and $h \in C^{n}([a, b], \mathbb{C})$, where $[a, b] \subset \mathbb{R}$, and let $\nu \geq 0: n=$ $\lceil\nu\rceil$. Assume that $h^{(i)}(a)=0, i=1, \ldots, n-1$. Then

$$
\left|\frac{1}{b-a} \int_{a}^{b} h(t) d t-f(a)\right| \leq \frac{\left\|D_{* a}^{\nu} h\right\|_{\infty,[a, b]}}{\Gamma(\nu+2)}(b-a)^{\nu}
$$

in particular when $h(t):=f(z(t)) z^{\prime}(t)$ and $\left.\left(f(z(t)) z^{\prime}(t)\right)^{(i)}\right|_{t=a}=0, i=1, \ldots n-1$, we get

$$
\begin{gathered}
\left|\frac{1}{b-a} \int_{\gamma_{u, w}} f(z) d z-f(u) z^{\prime}(a)\right|=\left|\frac{1}{b-a} \int_{a}^{b} f(z(t)) z^{\prime}(t) d t-f(z(a)) z^{\prime}(a)\right| \\
\leq \frac{\left\|D_{* a}^{\nu} f(z(t)) z^{\prime}(t)\right\|_{\infty,[a, b]}}{\Gamma(\nu+2)}(b-a)^{\nu}
\end{gathered}
$$

Proof. By Theorem 2.4.

The corresponding $\mathbb{C}$-Ostrowski type $L_{p}$ inequality follows:

Theorem 3.5. Let $p, q>1: \frac{1}{p}+\frac{1}{q}=1$, and $\nu>\frac{1}{q}, n=\lceil\nu\rceil$. Here $h \in C^{n}([a, b], \mathbb{C})$. Assume that $h^{(i)}(a)=0, i=1, \ldots, n-1$. Then

$$
\left|\frac{1}{b-a} \int_{a}^{b} h(t) d t-h(a)\right| \leq \frac{\left\|D_{* a}^{\nu} h\right\|_{L_{q}([a, b], \mathbb{C})}}{\Gamma(\nu)(p(\nu-1)+1)^{\frac{1}{p}}\left(\nu+\frac{1}{p}\right)}(b-a)^{\nu-\frac{1}{q}},
$$


in particular when $h(t):=f(z(t)) z^{\prime}(t)$ and $\left.\left(f(z(t)) z^{\prime}(t)\right)^{(i)}\right|_{t=a}=0, i=1, \ldots n-1$, we get:

$$
\begin{aligned}
\left|\frac{1}{b-a} \int_{\gamma_{u, w}} f(z) d z-f(u) z^{\prime}(a)\right| & =\left|\frac{1}{b-a} \int_{a}^{b} f(z(t)) z^{\prime}(t) d t-f(z(a)) z^{\prime}(a)\right| \\
& \leq \frac{\left\|D_{* a}^{\nu}\left(f(z(t)) z^{\prime}(t)\right)\right\|_{L_{q}([a, b], \mathbb{C})}}{\Gamma(\nu)(p(\nu-1)+1)^{\frac{1}{p}}\left(\nu+\frac{1}{p}\right)}(b)^{\nu-\frac{1}{q}} .
\end{aligned}
$$

Proof. By Theorem 2.5.

It follows

Corollary 3.6. (to Theorem 3.5, case of $p=q=2$ ). We have that

$$
\left|\frac{1}{b-a} \int_{\gamma_{u, w}} f(z) d z-f(u) z^{\prime}(a)\right| \leq \frac{\left\|D_{* a}^{\nu}\left(f(z(t)) z^{\prime}(t)\right)\right\|_{L_{2}([a, b], \mathbb{C})}}{\Gamma(\nu) \sqrt{2 \nu-1}\left(\nu+\frac{1}{2}\right)}(b-a)^{\nu-\frac{1}{2}} .
$$

We continue with an $L_{1}$ fractional $\mathbb{C}-$ Ostrowski type inequality:

Theorem 3.7. Let $\nu \geq 1, n=\lceil\nu\rceil$. Assume that $h \in C^{n}([a, b], \mathbb{C})$, where

$$
h(t):=f(z(t)) z^{\prime}(t),
$$

and such that $h^{(i)}(a)=0, i=1, \ldots, n-1$. Then

$$
\left|\frac{1}{b-a} \int_{\gamma_{u, w}} f(z) d z-f(u) z^{\prime}(a)\right| \leq \frac{\left\|D_{* a}^{\nu}\left(f(z(t)) z^{\prime}(t)\right)\right\|_{L_{1}([a, b], \mathbb{C})}}{\Gamma(\nu+1)}(b-a)^{\nu-1} .
$$

Proof. By Theorem 2.7.

It follows a Poincaré like $\mathbb{C}$-fractional inequality:

Theorem 3.8. Let $p, q>1: \frac{1}{p}+\frac{1}{q}=1$, and $\nu>\frac{1}{q}, n=\lceil\nu\rceil$. Let $h \in C^{n}([a, b], \mathbb{C})$. Assume that $h^{(i)}(a)=0, i=1, \ldots, n-1$. Then

$$
\|h\|_{L_{q}([a, b], \mathbb{C})} \leq \frac{(b-a)^{\nu}\left\|D_{* a}^{\nu} h\right\|_{L_{q}([a, b], \mathbb{C})}}{\Gamma(\nu)(p(\nu-1)+1)^{\frac{1}{p}}(q \nu)^{\frac{1}{q}}},
$$

in particular when $h(t):=f(z(t)) z^{\prime}(t)$ and $\left.\left(f(z(t)) z^{\prime}(t)\right)^{(i)}\right|_{t=a}=0, i=1, \ldots n-1$, we get:

$$
\begin{gathered}
\left\|\frac{\left\|f(z(t)) z^{\prime}(t)\right\|_{L_{q}([a, b], \mathbb{C})}}{\Gamma(\nu)(p(\nu-1)+1)^{\frac{1}{p}}(q \nu)^{\frac{1}{q}}}\right\| D_{* a}^{\nu}\left(f(z(t)) z^{\prime}(t)\right) \|_{L_{q}([a, b], \mathbb{C})} .
\end{gathered}
$$

Proof. By Theorem 2.8.

The corresponding Sobolev like inequality follows: 
Theorem 3.9. All as in Theorem 3.8. Let $r>0$. Then

$$
\leq \frac{\left\|f(z(t)) z^{\prime}(t)\right\|_{L_{r}([a, b], \mathbb{C})}}{\Gamma(\nu)(p(\nu-1)+1)^{\frac{1}{p}}\left(r\left(\nu-\frac{1}{q}+\frac{1}{r}\right)+1\right)^{\frac{1}{r}}}\left\|D_{* a}^{\nu}\left(f(z(t)) z^{\prime}(t)\right)\right\|_{L_{q}([a, b], \mathbb{C})} .
$$

Proof. By Theorem 2.9.

We continue with an Opial type $\mathbb{C}$-fractional inequality

Theorem 3.10. Let $p, q>1: \frac{1}{p}+\frac{1}{q}=1$, and $\nu>\frac{1}{q}, n:=\lceil\nu\rceil, h \in C^{n}([a, b], \mathbb{C})$. Assume $h^{(k)}(a)=0, k=0,1, \ldots, n-1$. Then

$$
\begin{gathered}
\int_{a}^{x}|h(t)|\left|\left(D_{* a}^{\nu} h\right)(t)\right| d t \\
\leq \frac{(x-a)^{\nu-1+\frac{2}{p}}}{2^{\frac{1}{q}} \Gamma(\nu)((p(\nu-1)+1)(p(\nu-1)+2))^{\frac{1}{p}}}\left(\int_{a}^{x}\left|\left(D_{* a}^{\nu} h\right)(t)\right|^{q} d t\right)^{\frac{2}{q}},
\end{gathered}
$$

$\forall x \in[a, b]$, in particular when $h(t):=f(z(t)) z^{\prime}(t)$ and $\left.\left(f(z(t)) z^{\prime}(t)\right)^{(i)}\right|_{t=a}=0$, $i=1, \ldots n-1$, we get:

$$
\begin{gathered}
\int_{a}^{x}|f(z(t))|\left|\left(D_{* a}^{\nu}\left(f(z(t)) z^{\prime}(t)\right)\right)\right|\left|z^{\prime}(t)\right| d t \\
\leq \frac{(x-a)^{\nu-1+\frac{2}{p}}}{2^{\frac{1}{q}} \Gamma(\nu)((p(\nu-1)+1)(p(\nu-1)+2))^{\frac{1}{p}}}\left(\int_{a}^{x}\left|D_{* a}^{\nu}\left(f(z(t)) z^{\prime}(t)\right)\right|^{q} d t\right)^{\frac{2}{q}},
\end{gathered}
$$

$\forall x \in[a, b]$.

Proof. By Theorem 2.10.

We finish with Hilbert-Pachpatte left $\mathbb{C}$-fractional inequalities:

Theorem 3.11. Let $p, q>1: \frac{1}{p}+\frac{1}{q}=1$, and $\nu_{1}>\frac{1}{q}, \nu_{2}>\frac{1}{p}, n_{i}:=\left\lceil\nu_{i}\right\rceil, i=1,2$. Let $h_{i} \in C^{n_{i}}\left(\left[a_{i}, b_{i}\right], \mathbb{C}\right), i=1,2$. Assume $h_{i}^{\left(k_{i}\right)}\left(a_{i}\right)=0, \quad k_{i}=0,1, \ldots, n_{i}-1 ; i=1,2$. Then

$$
\begin{gathered}
\int_{a_{1}}^{b_{1}} \int_{a_{2}}^{b_{2}} \frac{\left|h_{1}\left(t_{1}\right)\right|\left|h_{2}\left(t_{2}\right)\right| d t_{1} d t_{2}}{\left(\frac{\left(t_{1}-a_{1}\right)^{p\left(\nu_{1}-1\right)+1}}{p\left(p\left(\nu_{1}-1\right)+1\right)}+\frac{\left(t_{2}-a_{2}\right)^{q\left(\nu_{2}-1\right)+1}}{q\left(q\left(\nu_{2}-1\right)+1\right)}\right)} \\
\leq \frac{\left(b_{1}-a_{1}\right)\left(b_{2}-a_{2}\right)}{\Gamma\left(\nu_{1}\right) \Gamma\left(\nu_{2}\right)}\left\|D_{* a_{1}}^{\nu_{1}} h_{1}\right\|_{L_{q}\left(\left[a_{1}, b_{1}\right], \mathbb{C}\right)}\left\|D_{* a_{2}}^{\nu_{2}} h_{2}\right\|_{L_{p}\left(\left[a_{2}, b_{2}\right], \mathbb{C}\right)},
\end{gathered}
$$

in particular when $h_{1}\left(t_{1}\right):=f_{1}\left(z_{1}\left(t_{1}\right)\right) z_{1}^{\prime}\left(t_{1}\right)$ and $h_{2}\left(t_{2}\right):=f_{2}\left(z_{2}\left(t_{2}\right)\right) z_{2}^{\prime}\left(t_{2}\right)$, with $h_{i}^{\left(k_{i}\right)}\left(a_{i}\right)=0, k_{i}=0,1, \ldots n_{i}-1 ; i=1,2$, we get:

$$
\begin{gathered}
\int_{a_{1}}^{b_{1}} \int_{a_{2}}^{b_{2}} \frac{\left|f_{1}\left(z_{1}\left(t_{1}\right)\right) z_{1}^{\prime}\left(t_{1}\right)\right|\left|f_{2}\left(z_{2}\left(t_{2}\right)\right) z_{2}^{\prime}\left(t_{2}\right)\right| d t_{1} d t_{2}}{\left(\frac{\left(t_{1}-a_{1}\right)^{p\left(\nu_{1}-1\right)+1}}{p\left(p\left(\nu_{1}-1\right)+1\right)}+\frac{\left(t_{2}-a_{2}\right)^{q\left(\nu_{2}-1\right)+1}}{q\left(q\left(\nu_{2}-1\right)+1\right)}\right)} \leq \frac{\left(b_{1}-a_{1}\right)\left(b_{2}-a_{2}\right)}{\Gamma\left(\nu_{1}\right) \Gamma\left(\nu_{2}\right)} . \\
\left\|D_{* a_{1}}^{\nu_{1}}\left(f_{1}\left(z_{1}\left(t_{1}\right)\right) z_{1}^{\prime}\left(t_{1}\right)\right)\right\|_{L_{q}\left(\left[a_{1}, b_{1}\right], \mathbb{C}\right)}\left\|D_{* a_{2}}^{\nu_{2}}\left(f_{2}\left(z_{2}\left(t_{2}\right)\right) z_{2}^{\prime}\left(t_{2}\right)\right)\right\|_{L_{p}\left(\left[a_{2}, b_{2}\right], \mathbb{C}\right)} .
\end{gathered}
$$


Proof. By Theorem 2.11.

\section{References}

[1] Anastassiou, G., A strong fractional calculus theory for Banach space valued functions, Nonlinear Functional Analysis and Applications, 22(2017), no. 3, 495-524.

[2] Anastassiou, G., Complex Opial type inequalities, Romanian J. of Math. \& CS, 9(2019), no. 2, 93-97.

[3] Dragomir, S.S., An extension of Ostrowski inequality to the complex integral, RGMIA Res. Rep. Coll., 21(2018), Art 112, 17 pp.

[4] Volintiru, C., A proof of the fundamental theorem of Calculus using Hausdorff measures, Real Analysis Exchange, 26(2000/2001), no. 1, 381-390.

George A. Anastassiou

Department of Mathematical Sciences,

University of Memphis,

Memphis, TN 38152, U.S.A.

e-mail: ganastss@memphis.edu 